\title{
GEOMETRIC PROGRESSIONS IN SYNDETIC SETS
}

\author{
BHUWANESH RAO PATIL
}

\begin{abstract}
In order to investigate multiplicative structures in additively large sets, Beiglböck et al. raised a significant open question as to whether or not every subset of the natural numbers with bounded gaps (syndetic set) contains arbitrarily long geometric progressions. A result of Erdős implies that syndetic sets contain a 2-term geometric progression with integer common ratio, but we still do not know if they contain such a progression with common ratio being perfect square. In this article, we prove that for each $k \in \mathbb{N}$, a syndetic set contains 2-term geometric progressions with common ratios of the form $n^{k} r_{1}$ and $p^{k} r_{2}$, where $p \in \mathbb{P}$ (the set of primes), $n$ is a composite number, $r_{1} \equiv 1(\bmod n), r_{2} \equiv 1(\bmod p)$ and $r_{1}, r_{2} \in \mathbb{N}$. We also show that 2 -syndetic sets (sets with bounded gap two) contain infinitely many 2-term geometric progressions with their respective common ratios being perfect squares.
\end{abstract}

\section{INTRODUCTION}

Previous research (e.g. [1], [2], [3] and [7]) establishes that sets which are large in any of several multiplicative senses must have substantial additive structure. For example, a multiplicatively piecewise syndetic $\operatorname{set}^{1}$ in $\mathbb{N}$ must contain arbitrarily long arithmetic progressions [1, Theorem 1.3]. However, very little appears to be known as the existence of multplicative structures in "additively large" sets. If we define "additive largeness" as having positive upper asymptotic density ${ }^{2}$, then one can observe that there are additively large sets that do not contain three term geometric progressions. For instance, the set of square-free numbers is additively large, because it has positive upper asymptotic density, but does not contain a configuration of the form $\left\{x, x r^{2}\right\}$. One may still ask if the property of containing multiplicative structures holds for interesting classes of sets that are additively large. This brings us to the following definition:

Definition 1.1 (Syndetic set). If $l \in \mathbb{N}$, then $A \subset \mathbb{N}$ is called l-syndetic set if $A$ has a non-empty intersection with every set of $l$ consecutive natural numbers. A subset of the natural numbers which is l-syndetic for some $l \in \mathbb{N}$, is known as a syndetic set.

An infinite arithmetic progression is the simplest example of a syndetic set. Plainly, syndetic sets have positive upper asymptotic density and thus are additively large. Beiglböck et al. [1] recognized the significance of looking for geometric progressions in syndetic sets in order to study multiplicative structures in additively large sets. They asked the following question.

Question 1. If $A$ is syndetic, do there exist $x, y \in \mathbb{N}$ such that $\left\{x, x y, x y^{2}\right\} \subset A$ ?

In recent work[5], Daniel Glasscock et al. gave some evidence towards an affirmative answer to this question by showing that many syndetic sets of dynamical origin contain arbitrarily long geometric progressions. The fact that syndetic sets contain 2-term geometric progression with integer common ratio is a consequence

\footnotetext{
${ }^{1}$ For definition of multiplicatively piecewise syndetic set, see [1].

${ }^{2}$ Upper asymptotic density of a set $A \subset \mathbb{N}$ is defined by $\bar{d}(A):=\lim \sup \frac{|A \cap[1, n]|}{n}$
} 
of the following propositions, namely Proposition 1.2 for dense sets and Proposition 1.4 for additively piecewise syndetic sets.

Proposition 1.2 (Erdös [4]). Suppose that $A$ is a subset of natural numbers such that lower asymptotic density $\underline{d}(A):=\liminf \frac{|A \cap[1, n]|}{n}>0$. Then $A$ contains a 2 -term geometric progression with integer common ratio.

Definition 1.3 (Additively piecewise syndetic set). Let $A \subset \mathbb{N}$. Then $A$ is called additively piecewise syndetic set if there exists $l \in \mathbb{N}$ such that for every $n \in \mathbb{N}$, there is a sequence $\left(x_{i}\right)_{i=1}^{n}$ in $A$ satisfying $0<x_{i+1}-x_{i} \leq l \forall i \in[1, n-1]$. For example, every syndetic set is additively piecewise syndetic set.

Proposition 1.4. [1, Corollary 2.17] If $A$ is an additively piecewise syndetic set, then there exists a sequence $\left(y_{n}\right)_{n=1}^{\infty}$ in $\mathbb{N} \backslash\{1\}$ such that for each $n \in \mathbb{N}, y_{n+1} \equiv 1$ $\left(\bmod \prod_{i=1}^{n} y_{i}\right)$ and $\prod_{i=1}^{n} y_{i} \in A$.

In other words, an additively piecewise syndetic set contains configurations of the type $\{x, x y\}$ for some $x, y \in \mathbb{N}$. But there exists some additively piecewise syndetic set which does not contain configurations of the type $\left\{x, x y, x y^{2}\right\}$ with $x, y \in \mathbb{N}$. One can get this type of set inside the collection of thick sets where a thick set is a subset of the natural numbers containing arbitrarily long intervals in $\mathbb{N}$. Using the fact that every thick set is additively piecewise syndetic, Proposition 1.5 guarantees the existence of a piecewise syndetic set not containing configurations of the type $\left\{x, x y, x y^{2}\right\}$ with $x, y \in \mathbb{N}$.

Proposition 1.5. [1, Theorem 3.5] There is a thick subset $A$ of $\mathbb{N}$ such that there do not exist $a \in A$ and $r \in \mathbb{Q} \backslash\{1\}$ such that $a r \in A$ and $a r^{2} \in A$.

The following weaker version of Question 1 is still an open question.

Question 2. If $A \subset \mathbb{N}$ is syndetic, do there exist $x, y \in \mathbb{N}$ such that $\left\{x, x y^{2}\right\} \subset A$ ?

Our first result relates to the above question and gives more information about 2 -term geometric progressions with integer common ratios in syndetic sets.

Theorem 1.6. Let $k \in \mathbb{N}$ and $H_{0} \in\{\mathbb{P}, \mathbb{N} \backslash \mathbb{P}\}$. Then any syndetic set contains a 2 -term geometric progressions with common ratio $n^{k} r$ for some $n \in H_{0} \backslash\{1\}$ and $r \in \mathbb{N}$ with $r \equiv 1(\bmod n)$.

The next result of this paper confirms an affirmative answer to Question 2 in the case of 2-syndetic sets.

Theorem 1.7. A 2-syndetic set contains infinitely many 2-term geometric progressions whose common ratios are perfect squares.

The proof of Theorem 1.6 uses Chinese remainder theorem extensively. In Section 2, we show that generating pairwise prime sets [see Definition 2.1] in a syndetic set is enough for finding configurations as required in Theorem 1.6. Section 3 describes about Triveni triplets [see Definition 3.1] to understand pairwise prime subsets of a syndetic set. Zorn's lemma guarantees the existence of Triveni triplets of order one with respect to a given syndetic set and then repeated use of Chinese remainder theorem at various stages generates Triveni triplets of higher order. Using these observations, Section 3.3 explains the proof of Theorem 1.6. Section 4 describes the proof of Theorem 1.7 by producing infinitely many explicit geometric progressions.

Notation. Let $\mathbb{Q}, \mathbb{N}, \mathbb{P}$ and $\mathbb{Z}$ denote, respectively, the set of rational numbers, the set of positive integers, the set of prime numbers and the set of integers. For $k \in \mathbb{N}$ and $H \subset \mathbb{N}, \mathcal{S}_{k, l, H}$ denotes the collection of $l$-syndetic sets that contain a 
configuration of the form $\left\{x, x n^{k} r\right\}$, where $n \in H, r \in \mathbb{N}$ satisfying $r \equiv 1(\bmod n)$. Let $[a, b]:=\{x \in \mathbb{Z}: a \leq x \leq b\}$. Let $\subsetneq$ denote the "proper subset of". For $A \subset \mathbb{N}$ and $x \in \mathbb{N}, x A=A x:=\{n x: n \in A\}$.

\section{Chinese Remainder theorem and Syndetic Sets}

Definition 2.1 (Pairwise prime set). Let $B \subset \mathbb{N}$. Then $B$ is called a pairwise prime set if $\operatorname{gcd}(a, b)=1 \forall a, b \in B$ with $a \neq b$.

Using the Chinese Remainder Theorem[8], the next lemma helps us to prove Theorem 1.6 in the case of syndetic sets containing pairwise prime sets with arbitrarily large cardinalities.

Lemma 2.2. Let $h \in \mathbb{N}$, let $m_{1}, m_{2}, \ldots, m_{h}$ be pairwise co-prime integers in $\mathbb{N}$ and let $t_{1}, t_{2}, \ldots, t_{h}$ be arbitrary elements in $\mathbb{N} \cup\{0\}$. Then $\exists u_{0} \in \mathbb{N}$ such that if $u_{t}=u_{0}+t\left(\prod_{i=1}^{h} m_{i}^{2}\right)$ with $t \in \mathbb{N}$, then there exists $\left(r_{i, t}\right)_{i=1}^{h}$ in $\mathbb{N}$ satisfying $u_{t}+t_{i}=r_{i, t} m_{i}$ and $r_{i, t} \equiv 1\left(\bmod m_{i}\right) \quad \forall i \in[1, h]$.

Proof. Consider the congruences $x+t_{i} \equiv m_{i}\left(\bmod m_{i}^{2}\right) \forall i \in[1, h]$.

Since $\left\{m_{i}: i \in[1, h]\right\}$ is a pairwise prime subset in $\mathbb{N}$ and $\left\{t_{i}: i \in[1, h]\right\} \subset$ $\mathbb{N} \cup\{0\}$, the Chinese remainder theorem ensures the existence of $u_{0} \in \mathbb{N}$ such that if $u_{t}=u_{0}+t w^{2}$ for $w:=\prod_{i=1}^{h} m_{i}$ and $t \in \mathbb{N}$, then $u_{t}+t_{i} \equiv m_{i}\left(\bmod m_{i}^{2}\right) \forall i \in[1, h]$. Therefore there exists a sequence $\left(r_{i, t}\right)_{i=1}^{h}$ in $\mathbb{N}$ such that $u_{t}+t_{i}=r_{i, t} m_{i}$ and $r_{i, t} \equiv 1$ $\left(\bmod m_{i}\right) \forall i \in[1, h]$.

From the above lemma, we get the following important corollary which will be used recursively in the proof of Theorem 1.6.

Corollary 2.3. Let $h, k \in \mathbb{N}, A \subset \mathbb{N}$ be an infinite set and $B \subset \mathbb{N}$ be a pairwise prime set such that $|B|=h$ and $u B \subset A$ for some $u \in \mathbb{N}$. If $H$ be a pairwise prime subset of $\mathbb{N}$ such that $|H|=|B|$ and $\operatorname{gcd}(a, b)=1 \forall a \in H, b \in B$, then at least one of the following is true.

(1) A contains a configuration of the type $\left\{x, x n^{k} r\right\}$ for some $x, r \in \mathbb{N}, n \in H$ and $r \equiv 1(\bmod n)$.

(2) There exists $z^{\prime} \in \mathbb{N}$ such that $u\left[z_{t}, z_{t}+h-1\right] \cap A=\varnothing \quad \forall t \in \mathbb{N}$ where $z_{t}=z^{\prime}+t\left(\prod_{x \in B} x^{2}\right)\left(\prod_{x \in H} x^{2 k}\right)$

Proof. Let $B=\left\{x_{1}, x_{2}, \cdots, x_{h}\right\}$. Since $|B|=|H|$, there exists a bijective map $f: B \rightarrow H$. Define $m_{i}:=x_{i} f\left(x_{i}\right)^{k} \forall i \in[1, h]$.

Since $H$ and $B$ are pairwise prime sets satisfying $\operatorname{gcd}(a, b)=1 \forall a \in H, b \in B$, we get that the set $\left\{m_{i}: i \in[1, h]\right\}$ is a pairwise prime set. So, Lemma 2.2 gives $z^{\prime} \in \mathbb{N}$ which satisfies the property that if $z_{t}=z^{\prime}+t\left(\prod_{i=1}^{h} m_{i}^{2}\right)$ for $t \in \mathbb{N}$, then there exists a sequence $\left(r_{i, t}\right)_{i=1}^{h}$ in $\mathbb{N}$ such that

$$
z_{t}+i-1=r_{i, t} m_{i} \quad \text { and } r_{i, t} \equiv 1 \quad\left(\bmod m_{i}\right) \forall i \in[1, h] .
$$

Since $u B \subset A$ for some $u \in \mathbb{N}$, we have $y_{i}:=u x_{i} \in A \forall i \in[1, h]$. Hence, applying the definitions of $y_{i}$ and $m_{i}$ in the equation (2.1), we have

$$
u z_{t}+u(i-1)=y_{i} f\left(x_{i}\right)^{k} r_{i, t} \text { and } r_{i, t} \equiv 1 \quad\left(\bmod f\left(x_{i}\right)\right) \forall i \in[1, h] .
$$

If $u\left[z_{t}, z_{t}+h-1\right] \cap A \neq \varnothing$ for some $t \in \mathbb{N}$, then $\exists j \in[1, h]$ such that $y_{j} f\left(x_{j}\right)^{k} r_{j, t} \in$ $A$ and $r_{j, t} \equiv 1\left(\bmod f\left(x_{j}\right)\right)$ where $y_{j} \in A$ and $f\left(x_{j}\right) \in H$. This completes the proof.

For any finite pairwise prime set $B$ and an infinite pairwise prime subset of $\mathbb{N}$ (say $H_{0}$ ), there exists pairwise prime set $H \subset H_{0}$ such that $|H|=|B|$ and $\operatorname{gcd}(a, b)=1 \forall a \in H, b \in B$. Then applying $u=1$ in Corollary 2.3 , we immediately get the following proposition. 
Proposition 2.4. Let $k, l \in \mathbb{N}, H_{0}$ be an infinite pairwise prime subset of $\mathbb{N}$ and $A$ be an l-syndetic set. If $B$ is a pairwise prime subset of $A$ such that $|B| \geq l$, then $A \in \mathcal{S}_{k, l, H_{0}}$.

If $H_{0}=\mathbb{P}$ or an infinite pairwise prime subset of the set of composite numbers, we get Theorem 1.6 for those $l$-syndetic sets which contain a pairwise prime set $B$ with $|B| \geq l$.

\section{TRIVENI TRIPLETS AND SYNDETIC SETS}

In the previous section, we observed that our proof of Theorem 1.6 depends on the study of pairwise prime subsets of syndetic sets. For better understanding of these pairwise prime sets, we define Triveni triplet as follows. For $l \in \mathbb{N}$ and $p \in \mathbb{P}$, denote

$$
\begin{gathered}
r(p, l):=\max \left\{t \in \mathbb{N} \cup\{0\}: p^{t} \leq 2 l+1\right\}, \\
T(l):=\left\{\prod_{p \in \mathbb{P} \cap[2,2 l+1]} p^{r_{p}}: 0 \leq r_{p} \leq r(p, l)\right\} .
\end{gathered}
$$

Definition 3.1 (Triveni triplet). Let $l, h \in \mathbb{N}, A \subset \mathbb{N}$ and $F \subset T(l) \backslash\{1\}$. Then $(F, h, l)$ is called a Triveni triplet with respect to the set $A$ if there exists a sequence of pairwise prime sets $\left(B_{u}\right)_{u \in F}$ such that

(1) $\left|B_{u}\right|=h$ and $u B_{u} \subset A$ for each $u \in F$

(2) For distinct $u, v \in F, \operatorname{gcd}(x, y)=1 \forall x \in B_{u}$ and $y \in B_{v}$.

Triveni triplets with respect to the set $A$ are called $A$-Triveni triplets. $|F|$ is called the order of the A-Triveni triplet $(F, h, l)$.

3.1. Triveni triplets of order one with respect to syndetic sets. One can produce Triveni triplets of order one with respect to most of the syndetic sets using Zorn's lemma[6].

Lemma 3.2. Let $r>0$ and $A \subset \mathbb{N}$. Let $\mathbb{M}_{r}$ be the collection of pairwise prime subsets $B$ of $\mathbb{N}$ satisfying $r B=\{r x: x \in B\} \subset A$. Then $\exists B_{r} \in \mathbb{M}_{r}$ such that if $C \supset B_{r}$ and $C \in \mathbb{M}_{r}$, then $C=B_{r}$. Here $B_{r}$ is a maximal element of $\mathbb{M}_{r}$.

Proof. Let $\alpha$ be a chain in the partially ordered set $\left(\mathbb{M}_{r}, \subset\right)$. Then the union of every elements of $\alpha$ belongs to the set $\mathbb{M}_{r}$. Hence, Zorn's lemma guarantees the existence of a maximal element.

In the above lemma, $B_{r}$ may be an empty set. But certainly $B_{r} \neq \varnothing$ for some $r \in \mathbb{N}$. In particular, $B_{1} \neq \varnothing$. The next proposition deals with the existence of an infinite pairwise prime set $B_{r}$ for some $r \in[1, l]$ with respect to those $(2 l+1)$ syndetic sets which do not contain at least two elements of $x \mathbb{N}$ for each $x \in \mathbb{N}$.

Proposition 3.3. Let $A$ be a $(2 l+1)$-syndetic set with $|x \mathbb{N} \backslash A| \geq 2 \forall x \in \mathbb{N}$. Then $\exists d \in[1, l]$ and an infinite pairwise prime set $B \subset \mathbb{N}$ such that $d B \subset A$.

Proof. Let $B_{r}$ be a maximal pairwise prime set satisfying $r B_{r} \subset A \backslash[1, l]$ for each $r \in[1, l]$. Lemma 3.2 assures the existence of such $B_{r}$.

By way of contradiction, assume that $B_{r}$ is finite for each $r \in[1, l]$. Define

$$
C:=\bigcup_{r=1}^{l} r B_{r}=\left\{u_{1}, u_{2}, \ldots, u_{m}\right\} \subset A \text { and } t:=\prod_{i=1}^{m} u_{i} .
$$

Clearly, $C \neq \varnothing$ and $t>l$ as $B_{1} \neq \varnothing$. Since $|t \mathbb{N} \backslash A| \geq 2, \exists s \in \mathbb{N} \backslash\{1\}$ such that st $\notin A$. Then the $(2 l+1)$-syndeticity of $A$ ensures that $z_{j} \in A$ for some $j \in[-l, l] \backslash 0$ where $z_{i}:=s t+i \forall i \in[-l, l]$. Note that $z_{j}>t$ as $j \geq-l, s>1$ and $t>l$. Define

$$
d:=\max \left\{r \in[1, l]: B_{r} \neq \varnothing \text { and } r \mid j\right\} .
$$


Clearly, $d$ is well define as $B_{1} \neq \varnothing$. Since $d x$ divides $t$ for all $x \in B_{d}$ and $z_{j}>t$, we have $z_{j}>d x \forall x \in B_{d}$.

Let $x \in B_{d}$ and $d^{\prime}=\operatorname{gcd}\left(d x, z_{j}\right)$. By the definition of $t, d x \mid t$. It follows that $d^{\prime}=\operatorname{gcd}(d x, j)$. Using $x \in B_{d}$ and $d^{\prime} \mid d x$, we get that the pairwise prime set $Z:=\left\{\frac{d x}{d^{\prime}}\right\} \subset \mathbb{N}$ satisfies $d^{\prime} Z \subset A \backslash[1, l]$ and so $B_{d^{\prime}} \neq \varnothing$. Since $d \mid j$ and $d^{\prime} \mid j$, the definition of $d$ gives $d \mid d^{\prime}$ and $d^{\prime} \leq d$. Hence $d=\operatorname{gcd}\left(d x, z_{j}\right)$.

Therefore, we get $z_{j} \in A$ such that $\operatorname{gcd}\left(d x, z_{j}\right)=d$ and $z_{j}>d x$ for each $x \in B_{d}$. It gives us pairwise prime set $Y=B_{d} \cup\left\{\frac{z_{j}}{d}\right\}$ satisfying $d Y \subset A \backslash[1, l]$ and $B_{d} \subsetneq Y$. This set $Y$ contradicts the maximality of $B_{d}$. So, we get a contradiction to the assumption that $B_{r}$ is finite for each $r \in[1, l]$. Hence, there exists $r \in[1, l]$ such that $B_{r}$ is an infinite pairwise prime set.

Therefore, Triveni triplets of order one can be found as a corollary of the above proposition in the following way.

Corollary 3.4. Let $k, l \in \mathbb{N}, H_{0}$ be an infinite pairwise prime subset of $\mathbb{N}$ and $A$ be $a(2 l+1)$-syndetic set such that $A \notin \mathcal{S}_{k, 2 l+1, H_{0}}$. Then there exists $d \in[2, l]$ such that $(\{d\}, h, l)$ is an $A$-Triveni triplet for each $h \in \mathbb{N}$.

Proof. Suppose for each $d \in[2, l]$, there exists $h_{d} \in \mathbb{N}$ such that $\left(\{d\}, h_{d}, l\right)$ is not an $A$-Triveni triplet. Applying Proposition 3.3, we get that either $A \supset x \mathbb{N} \backslash\{x y\}$ for some $x, y \in \mathbb{N}$ or there exists an infinite pairwise prime subset $B$ satisfying $B \subset A$. Therefore, Proposition 2.4 concludes the result.

3.2. Triveni triplets of higher order with respect to syndetic sets. Now we shall see a procedure for generating Triveni triplets of higher order with respect to syndetic sets. First we will prove some necessary results to demonstrate the procedure. Due to the next lemma, one can construct sets with arbitrary large cardinalities in which the gcd of any two distinct elements belongs to the set $T(l)$. Note that gcd of any two distinct elements in an interval with cardinality $(2 l+1)$ belongs to the set $T(l)$.

Lemma 3.5. For $l \in \mathbb{N}$, there exist an increasing function $c: \mathbb{N} \longrightarrow \mathbb{N}$ and $a$ strictly increasing sequence of positive integers $\left(x_{i}\right)_{i=1}^{\infty}$ such that $x \leq c(h)$ and $\operatorname{gcd}(x, y) \in T(l) \forall h \in \mathbb{N}, x, y \in S_{h}$ with $x \neq y$ where $S_{h}=\cup_{i=1}^{h}\left[x_{i}, x_{i}+2 l\right]$.

Proof. Define $x_{1}:=1$ and $c(1):=2 l+1$. Then $\operatorname{gcd}(a, b) \in T(l)$ for $a, b \in S_{1}$.

For given $h \in \mathbb{N}$, suppose there exist a sequence $\left(x_{i}\right)_{i=1}^{h}$ in $\mathbb{N}$ and $c(h) \in \mathbb{N}$ such that $a \leq c(h)$ and $\operatorname{gcd}(a, b) \in T(l) \forall a, b \in S_{h}$ with $a \neq b$. To complete the proof by induction, we need to generate $x_{h+1}$ and $c(h+1) \operatorname{such}$ that $\operatorname{gcd}\left(x, x_{h+1}+j\right) \in T(l)$, $x_{h}<x_{h+1} \leq c(h+1)-2 l$ and $c(h) \leq c(h+1) \forall x \in S_{h}$ and $j \in[0,2 l]$. For this purpose, we define

$$
\begin{gathered}
m:=\prod_{x \in S_{h}} x \text { and } Y:=\left\{p^{r}: p \in \mathbb{P}, p^{r} \mid m \text { and } p^{r+1} \nmid m\right\} \\
x_{h+1}:=1+\prod_{u \in Y} u \text { and } c(h+1):=\max \left\{2 l+x_{h+1}, c(h)\right\} .
\end{gathered}
$$

The definition of $c(h+1)$ ensures $x_{h+1} \leq c(h+1)-2 l$ and $c(h) \leq c(h+1)$. Since $x_{h}$ divides $m$, the definition of $x_{h+1}$ gives us $x_{h}<x_{h+1}$.

Let $x \in S_{h}$ and $j \in[0,2 l]$. To show that $\operatorname{gcd}\left(x, x_{h+1}+j\right) \in T(l)$, let $q$ be a prime divisor of $x$ such that $q^{r(q, l)+1} \mid x$. Since $x \in S_{h}$, it follows that $q^{r(q, l)+1} \mid m$ which is followed by $q^{r(q, l)+1} \mid u$ for some $u \in Y$. Then $x_{h+1}+j \equiv j+1\left(\bmod q^{r(q, l)+1}\right)$ by the definition of $x_{h+1}$. Hence, $q^{r(q, l)+1} \nmid x_{h+1}+j$ because of the fact that $j+1 \in[1,2 l+1]$ but $q^{r(q, l)+1}>2 l+1$ by the definition of $r(q, l)$. Hence $q^{r(q, l)+1} \nmid \operatorname{gcd}\left(x, x_{h+1}+j\right)$. Since $r(p, l)=0 \forall p \in \mathbb{P} \cup[2 l+2, \infty)$, we have $\operatorname{gcd}\left(x, x_{h+1}+j\right) \in T(l)$ by definition of $T(l)$. 
In the above lemma, the definition of the element $x_{h+1}$ is inspired by the application of the Chinese remainder theorem on the congruences $x \equiv 1(\bmod u) \forall u \in Y$. One can choose any positive integer which satisfies these congruences and is greater than 1 .

Corollary 3.6. There exists a map $m: \mathbb{N} \times \mathbb{N} \rightarrow \mathbb{N}$ such that for $h, l, n \in \mathbb{N}$, an interval $[n, n+m(h, l)]$ contains a set $S$ with the following properties.

(i) $S=\bigcup_{i=1}^{h}\left[x_{i}, x_{i}+2 l\right]$ for some strictly increasing sequence $\left(x_{i}\right)_{i=1}^{h}$ in $\mathbb{N}$.

(ii) $\operatorname{gcd}(a, b) \in T(l) \forall a, b \in S$ with $a \neq b$.

Proof. For given $l, h \in \mathbb{N}$, Lemma 3.5 gives a strictly increasing sequence $\left(y_{i}\right)_{i=1}^{h}$ in $\mathbb{N}$ and a positive integer $c(h)$ such that $a \leq c(h)$ and $\operatorname{gcd}(a, b) \in T(l) \forall a, b \in$ $R$ with $a \neq b$ where $R=\cup_{i=1}^{h}\left[y_{i}, y_{i}+2 l\right]$. Hence, define a map $m: \mathbb{N} \times \mathbb{N} \rightarrow \mathbb{N}$ such that $m(h, l):=L+c(h)$ where $L=\prod_{q \in \mathbb{P} \cap[2, c(h)]} q^{r(q, l)+1}$.

Let $n \in \mathbb{N}$ and $a_{0} \in[n+1, n+L]$ be an integer divisible by $L$. Then we shall show that the set $S:=\left\{a_{0}+u: u \in R\right\}$ satisfies $S \subset[n, n+m(h, l)]$ along with the properties (i) and (ii) given in the statement of Corollary (i). By construction, one can note that property (i) is obvious. Using the fact that $a \leq c(h) \forall a \in R$ and $a_{0} \in[n+1, n+L]$, we get that $S \subset[n, n+m(h, l)]$.

To prove property (ii), Let $v_{1}, v_{2} \in S$ with $v_{1} \neq v_{2}$. Then for each $i \in\{1,2\}$, $v_{i}=a_{0}+u_{i}$ for some $u_{i} \in R$. By way of contradiction, assume that $p$ be a prime such that $p^{r(p, l)+1} \mid \operatorname{gcd}\left(v_{1}, v_{2}\right)$. Since $r(p, l) \in \mathbb{N} \cup\{0\}$, It follows that $p \mid u_{1}-u_{2}$. This implies $p \leq c(h)$ because $u_{1}, u_{2} \in[1, c(h)]$. Then, $p^{r(p, l)+1} \mid a_{0}$ by applying the definitions of $a_{0}$ and $L$. It follows that $p^{r(p, l)+1} \mid \operatorname{gcd}\left(u_{1}, u_{2}\right)$ by using assumption that $p^{r(p, l)+1} \mid \operatorname{gcd}\left(v_{1}, v_{2}\right)$. But, this contradicts the fact that $\operatorname{gcd}\left(u_{1}, u_{2}\right) \in T(l)$. So we get a contradiction to the assumption that $p^{r(p, l)+1} \mid \operatorname{gcd}\left(v_{1}, v_{2}\right)$.

Therefore, $p^{r(p, l)+1} \nmid \operatorname{gcd}\left(v_{1}, v_{2}\right)$ for each $p \in \mathbb{P}$ and hence $\operatorname{gcd}\left(v_{1}, v_{2}\right) \in T(l)$ because $r(p, l)=0 \forall p \in[2 l+2, \infty)$.

The next lemma solves a Diophantine problem using Chinese remainder theorem. Define $C(l):=(2 l+1)^{2 l+1}$ and note that $u<C(l) \forall u \in T(l)$.

Lemma 3.7. Let $\left(a_{i}\right)_{i=1}^{n}$ and $\left(u_{i}\right)_{i=1}^{n}$ be sequences in $\mathbb{N} \cup\{0\}$ and $T(l)$ respectively. If $X=\left\{x_{1}, x_{2}, \cdots x_{n}\right\}$ be a pairwise prime subset of $\mathbb{N}$, then there exist $z \in \mathbb{N}$, a sequence $\left(r_{i}\right)_{i=1}^{n}$ in $[0, C(l)]$ and a sequence $\left(t_{i}\right)_{i=1}^{n}$ in $\mathbb{N}$ such that

$$
z+r_{i}=a_{i}+t_{i} x_{i} u_{i} \forall i \in[1, n] .
$$

Proof. Let $u=\operatorname{lcm}\left(u_{1}, u_{2}, \cdots, u_{n}\right)$. Then $u \in T(l)$ and so $u<C(l)$. For each $i \in[1, n]$, choose non-negative integers $r_{i}$ and $b_{i}$ such that $a_{i}=b_{i} u+r_{i}$ and $0 \leq r_{i}<u$. Note that $r_{i} \in[0, C(l)]$ as $u<C(l)$. Since $X$ is a pairwise prime set, the Chinese remainder theorem gives $b \in \mathbb{N}$ and a sequence $\left(v_{i}\right)_{i=1}^{n}$ in $\mathbb{N}$ such that $b=b_{i}+v_{i} x_{i} \forall i \in[1, n]$. Hence, $t_{i}:=\frac{v_{i} u}{u_{i}}$ and $z:=b u$ satisfy the required equation in $(3.1)$.

Using the above corollaries and lemmas, the next two propositions demonstrate the complete procedure to generate Triveni triplets of higher order with respect to syndetic sets. Define the map $\Lambda: \mathbb{N} \times \mathbb{N} \rightarrow \mathbb{N}$ such that

$$
\Lambda(h, l)=m(h, l)+2 C(l) \forall h, l \in \mathbb{N}
$$

where the map $m: \mathbb{N} \times \mathbb{N} \rightarrow \mathbb{N}$ is taken from Corollary 3.6. For $l \in \mathbb{N}$ and $F \subset T(l)$, define

$$
D(l):=|T(l)| \text { and } \operatorname{Mul}(F):=\{v \in T(l): \exists u \in F \text { such that } u \mid v\} .
$$


Proposition 3.8. Let $k, l, h \in \mathbb{N}, A$ be $a(2 l+1)$-syndetic set and $H_{0}$ be an infinite pairwise prime subset of $\mathbb{N}$. If $(F, \Lambda(D(l) h, l), l)$ is an $A$-Triveni triplet and $A \notin \mathcal{S}_{k, 2 l+1, H_{0}}$, then $\exists w \in T(l) \backslash \operatorname{Mul}(F)$ and a pairwise prime set $C_{w}$ such that $\left|C_{w}\right|=h$ and $w C_{w} \subset A$.

Proof. Since $(F, \Lambda(D(l) h, l), l)$ is an $A$-Triveni triplet, there exists a sequence of pairwise prime sets $\left(B_{u}\right)_{u \in F}$ satisfying $\left|B_{u}\right|=\Lambda(D(l) h, l)$ and $u B_{u} \subset A$ for each $u \in F$ such that for distinct $u, v \in F$,

$$
\operatorname{gcd}(x, y)=1 \forall x \in B_{u} \text { and } y \in B_{v} .
$$

Let $B=\cup_{u \in F} B_{u}$. Since $H_{0}$ is an infinite pairwise prime set, there exists a sequence of pairwise prime subsets of $H_{0}\left(\right.$ say $\left.\left(H_{u}\right)_{u \in F}\right)$ satisfying $\left|B_{u}\right|=\left|H_{u}\right|$ $\forall u \in F$ such that for distinct $u, v \in F$,

$$
\operatorname{gcd}(x, y)=\operatorname{gcd}(x, b)=1 \forall x \in H_{u}, y \in H_{v} \text { and } b \in B .
$$

Given that $A$ is a $(2 l+1)$-syndetic set satisfying $A \notin \mathcal{S}_{k, 2 l+1, H_{0}}$. Applying Corollary 2.3, $\exists$ a sequence $\left(z_{u}\right)_{u \in F}$ in $\mathbb{N}$ such that if $z_{u, t}=z_{u}+t\left(\prod_{x \in B_{u}} x^{2}\right)\left(\prod_{x \in H_{u}} x^{2 k}\right)$ with $t \in \mathbb{N}$, then

$$
u\left[z_{u, t}, z_{u, t}+\Lambda(D(l) h, l)-1\right] \cap A=\varnothing \forall t \in \mathbb{N}, u \in F .
$$

By Lemma 3.7, there exist sequences $\left(r_{u}\right)_{u \in F}$ in $[0, C(l)],\left(t_{u}\right)_{u \in F}$ in $\mathbb{N}$ and $z \in \mathbb{N}$ such that $z+r_{u}=u z_{u, t_{u}} \forall u \in F$. Then equation (3.2) guarantees that $u \mathbb{N} \cap A \cap[z+$ $\left.r_{u}, z+r_{u}+u \Lambda(D(l) h, l)-u\right]=\varnothing \forall u \in F$. Since $r_{u} \in[0, C(l)]$ and $1<u<C(l)$ $\forall u \in F$, it follows that $u \mathbb{N} \cap A \cap I=\varnothing \forall u \in F$ where

$$
I=[z+C(l), z+\Lambda(D(l) h, l)-C(l)]=[z+C(l), z+C(l)+m(D(l) h, l)] .
$$

Hence, Corollary 3.6 gives us a sequence of intervals in $I$ (say $\left(S_{i}\right)_{i=1}^{D(l) h}$ ) such that

(a) $\left|S_{i}\right|=2 l+1$ and $A \cap S_{i} \cap u \mathbb{N}=\varnothing \forall i \in[1, D(l) h], u \in F$,

(b) $v_{1} \in S_{i}, v_{2} \in S_{j}$ and $v_{1} \neq v_{2}$ for $i, j \in[1, D(l) h] \Rightarrow \operatorname{gcd}\left(v_{1}, v_{2}\right) \in T(l)$.

Since $A$ is $(2 l+1)$-syndetic set, there exists a sequence $\left(s_{i}\right)_{i=1}^{D(l) h}$ such that $s_{i} \in S_{i} \cap A$ and $\operatorname{gcd}\left(s_{i}, s_{j}\right) \in T(l) \backslash \operatorname{Mul}(F)$ for $i \neq j$. Define

$$
g_{i}:=\max \left\{u \in T(l) \backslash \operatorname{Mul}(F): u \mid s_{i}\right\} \forall i \in[1, D(l) h] .
$$

Using $|T(l)|=D(l)$, there exist $J \subset[1, D(l) h]$ and $w \in T(l) \backslash \operatorname{Mul}(F)$ such that $|J| \geq h$ and $g_{j}=w \forall j \in J$. Set $C_{w}:=\left\{\frac{s_{j}}{w}: j \in J\right\}$.

Since $g_{j}=w \forall j \in J$ and $\operatorname{gcd}\left(s_{j_{1}}, s_{j_{2}}\right) \in T(l) \backslash M u l(F) \forall j_{1}, j_{2} \in J$ with $j_{1} \neq j_{2}$, we have $\operatorname{gcd}\left(s_{j_{1}}, s_{j_{2}}\right)=w \forall j_{1}, j_{2} \in J$ with $j_{1} \neq j_{2}$. Therefore, $C_{w}$ is a pairwise prime set and $w C_{w}=\left\{s_{j}: j \in J\right\} \subset A$.

Proposition 3.9. Suppose that $k, l \in \mathbb{N}, H_{0}$ be an infinite pairwise prime subset of $\mathbb{N}$ and $A$ be a $(2 l+1)$-syndetic set with $A \notin \mathcal{S}_{k, 2 l+1, H_{0}}$. If $(F, h, l)$ is an $A$-Triveni triplet for each $h \in \mathbb{N}$, then $\exists F^{\prime} \subset T(l) \backslash\{1\}$ with $F \subsetneq F^{\prime}$ such that $\left(F^{\prime}, h, l\right)$ is an $A$-Triveni triplet for each $h \in \mathbb{N}$.

Proof. Let $k_{0} \in \mathbb{N} \backslash[1,2 l+1]$. Since $\left(F, k_{0}, l\right)$ is an $A$-Triveni triplet, there exists a sequence of pairwise prime sets $\left(B_{u}\right)_{u \in F}$ such that for every distinct $u, u_{1} \in F$, $\left|B_{u}\right|=k_{0}$ and $\operatorname{gcd}(x, y)=1 \forall x \in B_{u}, y \in B_{u_{1}}$. Let $B:=\cup_{u \in F} B_{u}, \alpha:=\{p \in \mathbb{P}: p \mid$ $x$ for some $x \in B\}$ and $W$ be the cardinality of $\alpha$.

Since $A$ is a $(2 l+1)$-syndetic set with $A \notin \mathcal{S}_{k, 2 l+1, H_{0}}$ and $(F, h, l)$ is an $A$-Triveni triplet for each $h \in \mathbb{N}$, Proposition 3.8 guarantees the existence of an element $v \in T(l) \backslash$ $\operatorname{Mul}(F)$ and a pairwise prime set $C_{v}$ such that $\left|C_{v}\right|=W+k_{0}$ and $v C_{v} \subset A$. Also, Proposition 2.4 ensures $v \neq 1$. Using the fact that $\alpha \subset \mathbb{P}$ with $|\alpha|=W$ and $C_{v}$ is a pairwise prime set of cardinality $W+k_{0}$, we get a pairwise prime set $B_{v} \subset C_{v}$ such that $\left|B_{v}\right|=k_{0}$ and $\operatorname{gcd}(p, b)=1 \forall p \in \alpha, b \in B_{v}$. Then $\operatorname{gcd}(a, b)=1 \forall a \in B, b \in B_{v}$ because elements of the pairwise prime set $B$ are made from primes in $\alpha$. Moreover 
BHUWANESH RAO PATIL

$v B_{v} \subset A$, because $B_{v} \subset C_{v}$ and $v C_{v} \subset A$. Since $\left(F, k_{0}, l\right)$ is an $A$-Triveni triplet, therefore, $\left(F_{k_{0}}, k_{0}, l\right)$ is also an $A$-Triveni triplet with $F_{k_{0}}=F \cup\{v\} \subset T(l) \backslash\{1\}$.

Here we constructed a sequence $\left(F_{n}\right)_{n \in \mathbb{N}}$ such that $F \subsetneq F_{n} \subset T(l) \backslash\{1\}$ and $\left(F_{n}, n, l\right)$ is an $A$-Triveni triplet $\forall n \in \mathbb{N}$. Since $|T(l)|<\infty$, there exist subsequence $\left(F_{n_{t}}\right)_{t \in \mathbb{N}}$ of $\left(F_{n}\right)_{n \in \mathbb{N}}$ and set $F^{\prime}$ with $F \subsetneq F^{\prime} \subset T(l) \backslash\{1\}$ such that $F_{n_{t}}=F^{\prime} \forall$ $t \in \mathbb{N}$. Hence, $\left(F^{\prime}, t, l\right)$ is an $A$-Triveni triplet for each $t \in \mathbb{N}$.

The combination of Corollary 3.4 and Proposition 3.9 generates Triveni triplets of various orders with respect to those syndetic sets which do not contain configurations of the form $\left\{x, x n^{k} r\right\}$ where $r \in \mathbb{N}, n \in H_{0}$ with $r \equiv 1(\bmod n)$. Using these observations, we will now see the proof of Theorem 1.6.

3.3. Proof of Theorem 1.6. Let $H_{0}=\mathbb{P}$ or $H_{0}$ be an infinite pairwise prime subset of the set of composite numbers. Since $A$ is a syndetic set, there exists $l \in \mathbb{N}$ such that $A$ is a $(2 l+1)$-syndetic set.

For $k \in \mathbb{N}$, if possible assume that $A \notin \mathcal{S}_{k, 2 l+1, H_{0}}$. Then Corollary 3.4 gives the existence of an integer $d \in[2, l]$ such that $(\{d\}, h, l)$ is an $A$-Triveni triplet for each $h \in \mathbb{N}$. Therefore, by Proposition 3.9, there exists a sequence $\left(F_{i}\right)_{i=0}^{\infty}$ such that

(a) for each $i \in[0, \infty)$ and $h \in \mathbb{N},\left(F_{i}, h, l\right)$ is an $A$-Triveni triplet,

(b) $\{d\}=F_{0} \subsetneq F_{1} \subsetneq F_{2} \subsetneq \cdots \subsetneq F_{i} \subsetneq \cdots \subset T(l)$.

Since $|T(l)|<\infty$, property (b) of sequence $\left(F_{i}\right)_{i=0}^{\infty}$ is a contradiction. Therefore, $A \in \mathcal{S}_{k, 2 l+1, H_{0}}$. In other words, the syndetic set $A$ contains configurations of the form $\left\{x, x n^{k} r\right\}$ where $r \in \mathbb{N}, n \in H_{0}$ satisfying $r \equiv 1(\bmod n)$.

\section{Proof of Theorem 1.7}

Now we are going to prove Theorem 1.7 by generating the required configurations using two different algorithms.

Lemma 4.1. A 2-syndetic set $S$ contains infinitely many configurations of the type $\left\{x, x r^{2}\right\}$ or infinitely many odd perfect squares.

Proof. Let $m \in \mathbb{N}$ be an odd integer. If $m^{2}+1 \notin S$, then the 2 -syndeticity of $S$ ensures that the odd perfect square $m^{2} \in S$. On the other hand, if $m^{2}+1 \in S$, then the identity $\left(2 m^{2}+1\right)^{2}-1=4 m^{2}\left(m^{2}+1\right)$ guarantees that $\left\{x, x r^{2}\right\} \subset S$ for $x=m^{2}+1$ and $r=2 m$ whenever $\left(2 m^{2}+1\right)^{2}-1 \in S$. For the case $\left(2 m^{2}+1\right)^{2}-1 \notin S$, the odd perfect square $\left(2 m^{2}+1\right)^{2} \in S$ due to the 2-syndeticity of $S$. Hence, infinitude of the odd integers completes the proof.

Lemma 4.2. If $y \in 2 \mathbb{N}+1$ and $S$ is a 2-syndetic set such that $y^{2}\left(y^{2}+2 i\right) \in S$ for each $i \in\{1,-1\}$, then $S$ contains a configuration of the type $\left\{x, x r^{2}\right\}$ with $x, r \in \mathbb{N} \backslash\{1\}$ and $x \geq \frac{y^{2}-1}{4}$.

Proof. If $y^{2}+2 i \in S$ for some $i \in\{1,-1\}$, then $\left\{x, x r^{2}\right\} \subset S$ for $x=y^{2}+2 i$ and $r=y$. On the other hand, if $y^{2}+2 i \notin S$ for each $i \in\{1,-1\}$, then $\left\{y^{2}-1, y^{2}+3\right\} \subset S$ due to 2 -syndeticity of the set $S$. Since $y$ is an odd integer, we get consecutive natural numbers $a_{y}$ and $b_{y}$ satisfying $4 a_{y}=y^{2}-1 \in S$ and $4 b_{y}=y^{2}+3 \in S$. Since $S$ is a 2-syndetic set, so it follows that one of $a_{y}$ and $b_{y}$ lies inside $S$. Hence, $S$ contains $\left\{a_{y}, 4 a_{y}\right\}$ or $\left\{b_{y}, 4 b_{y}\right\}$. Therefore, $S$ contains the configuration $\left\{x, x r^{2}\right\}$ for $x \in\left\{a_{y}, b_{y}\right\}$ and $r=2$.

Proof of Theorem 1.7 (first method). Let $S$ be a 2-syndetic set. By Lemma 4.1, it is enough to show that if $m^{2} \in S$ for some $m \in 2 \mathbb{N}+1$, then $S$ contains a configuration of the type $\left\{x, x r^{2}\right\}$ where $x, r \in \mathbb{N} \backslash\{1\}$ and $x \geq \frac{m-1}{4}$ 
Let $m \in \mathbb{N} \backslash\{1\}$ be an odd integer such that $m^{2} \in S$. If $m^{2} n^{2} \in S$ for some $n \in \mathbb{N} \backslash\{1\}$, then we have $\left\{x, x r^{2}\right\} \subset S$ for $x=m^{2}$ and $r=n$. If $m^{2} n^{2} \notin S$ for each $n \in \mathbb{N} \backslash\{1\}$, then the 2-syndeticity of $S$ ensures that

$$
m^{2} n^{2}-1 \in S \forall n \in \mathbb{N} \backslash\{1\} .
$$

Choose $z \in 2 \mathbb{N}+1$ satisfying $z^{2} \equiv 0(\bmod m)$. Define $u(z, i):=z^{2}+i$ for each $i \in\{-1,1\}$. Clearly $u(z, i)^{2} \equiv 1(\bmod m)$ for each $i \in\{-1,1\}$. Then, for each $i \in\{-1,1\}$, there exists $k_{u(z, i)} \in \mathbb{N} \backslash\{1\}$

$$
m k_{u(z, i)}+1=u(z, i)^{2} .
$$

Putting $n=k_{u(z, i)}$ in expression (4.1), we get $m^{2} k_{u(z, i)}^{2}-1 \in S$. Inserting the value of $k_{u(z, i)}$ from equation (4.2) in this, we have $m^{2} k_{u(z, i)}^{2}-1=\left(u(z, i)^{2}-2\right) u(z, i)^{2} \in$ $S$.

Therefore, $\left\{x, x r^{2}\right\} \subset S$ for $x=u(z, i)^{2}-2$ and $r=u(z, i)$ whenever $u(z, i)^{2}-2 \in$ $S$. For the remaining case $u(z, i)^{2}-2 \notin S \forall i \in\{-1,1\}$, the 2 -syndeticity of $S$ guarantees that

$$
u(z, i)^{2}-1=z^{2}\left(z^{2}+2 i\right) \in S \forall i \in\{1,-1\} .
$$

Therefore we complete the proof by taking $y=z$ in Lemma 4.2.

The above algorithm also guarantees a configuration of the form $\left\{x, x r^{2}\right\}$ with $r<x$ inside syndetic sets containing the fourth power of some odd integer. For this, take $m$ to be an odd perfect square and choose $z=\sqrt{m}$ for some $i \in\{1,-1\}$ in the algorithm in the first method. The next algorithm generates a configuration of the form $\left\{x, x r^{2}\right\}$ inside syndetic sets with the condition $r>x$ using the identity in the following lemma.

Lemma 4.3. For $a \in \mathbb{N}, a(4 a+3)^{2}+1=(a+1)(4 a+1)^{2}$.

Proof of Theorem 1.7 (second method). Let $S$ be a 2-syndetic set. If $\{a, a+1\} \subset S$ for infinitely many $a \in \mathbb{N}$, then applying the identity in Lemma 4.3 , we get that $\left\{x, x r^{2}\right\} \subset S$ for $(x, r)=(a,(4 a+3))$ or $(a+1,(4 a+1))$ for those $a^{\prime}$ s. On the other hand, $S$ contains an infinite arithmetic progression whenever $\{a, a+1\} \subset S$ for only finitely many $a \in \mathbb{N}$. Therefore, we finish the proof by using the fact that any infinite arithmetic progression contains an infinite geometric progression.

\section{REFERENCES}

[1] Mathias Beiglböck, Vitaly Bergelson, Neil Hindman, Dona Strauss, Multiplicative structures in additively large sets. J. Combin. Theory Ser. A 113 (2006), no. 7, 1219-1242.

[2] V Bergelson, Multiplicatively large sets and ergodic Ramsey theory, Israel J. Math. 148 (2005) 23-40.

[3] V Bergelson and D Glasscock, On the interplay between additive and multiplicative largeness and its combinatorial applications https://arxiv.org/pdf/1610.09771.pdfv2

[4] Paul Erdős, Note on Sequences of Integers No One of Which is Divisible By Any Other. J. London Math. Soc. 10 (1935), no. 2, 126-128.

[5] Daniel Glasscock, Andreas Koutsogiannis and Florian K. Richter, Multiplicative combinatorial properties of return time sets in minimal dynamical systems, To appear in Discrete and Continuous Dynamical Systems. https://arxiv.org/pdf/1809.08702.

[6] Paul R Halmos, Naive Set Theory, Undergrad. Texts in Math Springer-Verlag, New York, 1974

[7] N. Hindman, D. Strauss, Algebra in the Stone-Čech compactification. Theory and applications., Walter de Gruyter Co.(2012).

[8] M B Nathanson, Elementary methods in number theory. Graduate Texts in Mathematics, 195. Springer-Verlag, New York, 2000. xviii+513 pp.

Harish-Chandra Research Institute, HBNi, Chhatnag Road, Jhunsi, Allahabad - 211019, UtTar Pradesh, INDia. 